\title{
OPTIMIERENDE BETRIEBSMITTELEINSATZPLANUNG IM ÖFFENTLICHEN PERSONENNAHVERKEHR
}

\author{
Manfred Völker, HanseCom GmbH, Hamburg
}

Den wesentlichen Anteil an der Betriebsmitteleinsatzplanung in Unternehmen des öffentlichen Pərsonennahverkehrs (OPNV) stellt die Einsatzplanung von Fahrzeugen und Fahrpersonal dar. Diese sogenannte Fahr- und Dienstplanung ist heute ab einer gewissen Betriebsgröße nicht mehr ohne (optimierende) DV-Unterstützung denkbar. Eine solche Unterstützung bietet zum Beispiel das Programmsystem HOT. Ein Uberblick über Problemstellung und mathematische Verfahren in der Fahr-und Dienstplanung wurde in den Operations Research Proceedings 1986 gegeben. In jüngster Zeit ist das System HOT weiterentwickelt worden, sowohl die Benutzeroberfläche als auch die implementierten Algorithmen. In diesem Vortrag steht der in der Praxis erzielte Nutzen im Vordergrund.

Zunächst auf einem Großrechner realisiert, wurde HOT 1987 auf das Betriebssystem UNIX portiert. In seiner heutigen Version gliedert sich das System in die fünf Module Datenverwaltung, Sensitivizätsanalyse, Fahrzeug-Umlaufbiidung, Dienstplanbildung und Dienstreihenfolgenbildung. Bis auf die Datenverwaltung basieren alle Moduln auf Optimierungsalgorithmen. Die Benutzeroberfläche ist menügesteuert, die Dateneingabe erfolgt über Eingabemasken und Grafikoberflächen.

Der bei den HOT-Anwendern beobachtete Nutzen wird einerseits anhand des Einsparpotentials beim Fahrpersonal dargestellt. So ist der HOT-Anwender in der Lage, "auf Knopfdruck" die Änderung zahlreicher, betrieblicher Vorgaben (wie z.B. maximale Dienstdauer) in ihrer Kostenwirksamkeit zu prüfen. Die Einflußmöglichkeiten der Parameter werden erläutert. Die im Vortrag dargestellte Funktionalität der Dienstreihenfolgenbildung beinhaltet neben der optimalen Verteilung der Wochenarbeitszeit eine besonders benutzerfreundliche Oberfläche.

Andererseits wird über die Optimierung beim Fahrzeugeinsatz berichtet. Mit dem Modul Sensitivitätsanalyse wurden in der Praxis Einsparungen von mehr als einer Million D-Mark erzielt. In der Umlaufbildung wurden neue Eingriffsmöglichkeiten für den Anwender zur Steuerung des Optimierungsprozesses geschaffen. 